\title{
Multicore Fibre Fan-In/Fan-Out Device using Fibre Optic Collimators
}

\author{
Y. Jung, J. R. Hayes, S. U. Alam and D. J. Richardson \\ Optoelectronics Research Centre, University of Southampton, Southampton, UK \\ "Corresponding Authors: ymj@orc.soton.ac.uk
}

\begin{abstract}
We present a new approach in realizing multicore fibre fan-in/fan-out (FIFO) device. Using simple image demagnification by two fibre optic collimators a compact FIFO device for 4-core multicore fibre is fabricated with low crosstalk.
\end{abstract}

\section{Introduction}

One of the key optical components for space division multiplexed (SDM) transmission systems [1-3] is an efficient and stable SDM multiplexer/demultiplexer, which allows for individual spatial modes (or cores) to be accessed with minimal loss and crosstalk. In the case of multicore fibre (MCF), spatial multiplexers/demultiplexers are referred to as fan-in/fan-out (FIFO) devices and are used to efficiently couple light from individual single mode fibres to each core of the $M C$ and vice versa. Various configurations have been reported so far but the most common techniques use: 1) fused tapers [4], 2) 3D waveguides [5] and 3) free space optics [6]. Each of these approaches has its own merits, but it is still challenging to realize low crosstalk (XT) for high density MCFs having a small core pitch distance. In a fibre taper approach, for example, FIFO device can be effectively fabricated by tapering single mode fibre bundles but the mode field diameter (MFD) of each core enlarges during tapering, which can

(a)

a)

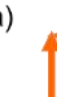

induce significant crosstalk between neighboring cores. In the case of the 3D waveguide approach, the maximum achievable refractive index change possible by direct femtosecond laser inscription is currently limited to about 0.007 and it is difficult to realize a low XT FIFO device with such a weakly confining waveguides. The free space optics based FIFO device shows a low insertion loss and XT but requires expensive custom optics and ultra-precise alignment, and represents the most expensive and bulky solution among the three approaches. Moreover, recent high density MCFs have square lattice core arrangements which is problematic as various of the FIFO fabrication methods cannot be applied to nonhexagonal lattice core arrangement (e.g. for fibre taper based FIFO, it is difficult to align and maintain the desired square lattice arrangement during the tapering and a special micro-hole arrayed glass platform needs to be used).

In this paper, we propose a compact and low XT FIFO device using commercially available fibre-optic collimator assemblies. Our approach

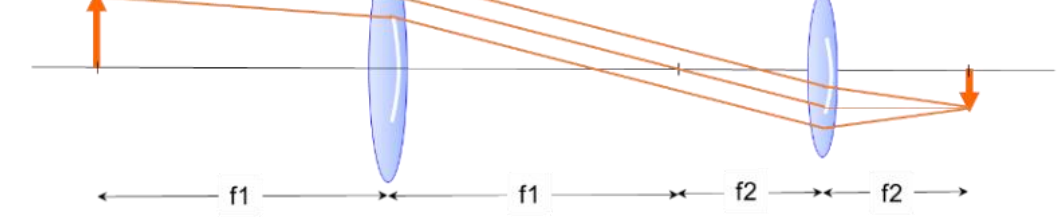

(c)

(b)

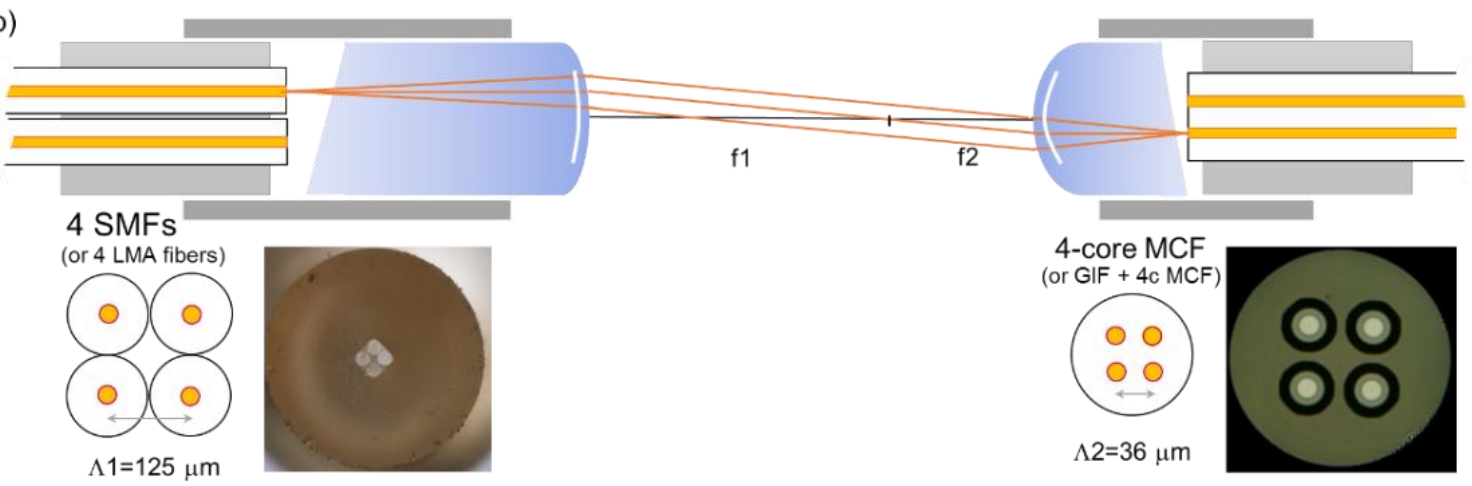

Fig. 1: (a) Geometry of image demagnification by two simple convex lenses with different focal lengths, (b) the analogous fibre optic collimator assembly for a compact FIFO device for a 4-core MCF and (c) the fully integrated device. 
is based on simple image demagnification using two micro-lens collimators having different focal lengths and can be readily implemented for most MCFs having either a square or hexagonal core arrangement. As an example we demonstrate a compact, proof of concept, FIFO device for 4core $\operatorname{MCF}(\Lambda=36 \mu \mathrm{m})$ which exhibits a moderate insertion loss $(<3.1 \mathrm{~dB})$ and low XT $(<-40 \mathrm{~dB})$.

\section{Working principle and fabrication of $4 \mathrm{C}-\mathrm{MCF}$ FIFO}

Figure 1(a) shows the basic geometry of image demagnification using two simple convex lenses. When an object is placed at the focal point of the first convex lens in a $4 f$-imaging system, a real, inverted and de-magnified image can be formed at the focal point behind the second convex lens. The demagnification ratio is determined by the ratio of the focal lengths of the two lenses (f2/f1) and can be used to convert the core pitch distance from the input SMFs to the output MCF to realize a FIFO device. For example, the square beam profile from 4 SMFs with $\Lambda 1=125 \mu \mathrm{m}$ can be converted to a similar square beam profile but with a reduced core pitch $(\Lambda 2=36 \mu \mathrm{m})$ at the image plane by choosing the appropriate focal lengths ratio of the two lenses i.e. $f 2 / f 1=36 / 125=0.29$ for low loss coupling. An analogous fibre optic collimator structure can be realized with two micro-optic lenses (e.g. C-lens or Grin lens) as shown in Fig. 1(b). By choosing an appropriate focal lengths of the two microlenses, a compact FIFO device can be easily realized with widely used fibre optic collimator assemblies. Note however that the MFD of the input fibres will also be reduced during the demagnification process by a factor of 0.29 and the subsequent MFD mismatch results in a significant coupling loss (3-4 dB) to the output 4cMCF. However, the coupling loss can be improved by employing thin cladding input fibres to reduce the demagnification ratio in conjunction with large mode area (LMA) fibres to enlarge the MFD of the input fibres.

In our experiment, four SMFs were first cleaved, inserted into a fibre ferrule with a square hole (dimension $=252 \times 252 \mu \mathrm{m}$ ) and positioned at the focal plane of the first C-lens $(f 1=4.77 \mathrm{~mm})$ to achieve an array of high quality collimated beams $[7,8]$. A low viscosity epoxy resin was applied inside the ferrule hole so that the surface tension of the epoxy helps to position the fibres adjacent to each other. As shown in Fig. 1b (left, bottom), 4 SMFs are closely packed with a core pitch distance of $125 \mu \mathrm{m}$. Next, another collimator was fabricated for the 4c-MCF using a shorter focal length C-lens $(f 2=1.87 \mathrm{~mm})$. Currently, the range of available $\mathrm{C}$-lens is somewhat limited and the focal length ratio used $(f 2 / f 1=1.87 / 4.77=0.39)$ is bigger than the required demagnification ratio (0.29). Therefore we have employed another core pitch adaptor using a graded index fibre lens to further adjust the de-magnification ratio of the object, which is detailed in ref. [9]. This all-fibre core pitch adaptor can be efficiently used for small core pitch conversion and fabricated by splicing a $1500 \mu \mathrm{m}$ segment of a graded index fibre (core diameter $=185 \mu \mathrm{m}$ ) to the 4c-MCF. Note that this all fibre core pitch adaptor can be used alone or together with other FIFO fabrication techniques (e.g. fused taper or free space optics) for precise control of the core pitch adaptation without significant XT increase.

Next, these two collimators were mounted on a multi-axis precision micro-positioner (offering translation, tilt and rotation adjustment) to align the collimators. After obtaining the maximum coupling efficiency, the collimator assemblies are fixed with low shrinkage UV curable epoxy. A final packaged 4c-MCF FIFO device is shown in Fig. 1(c).

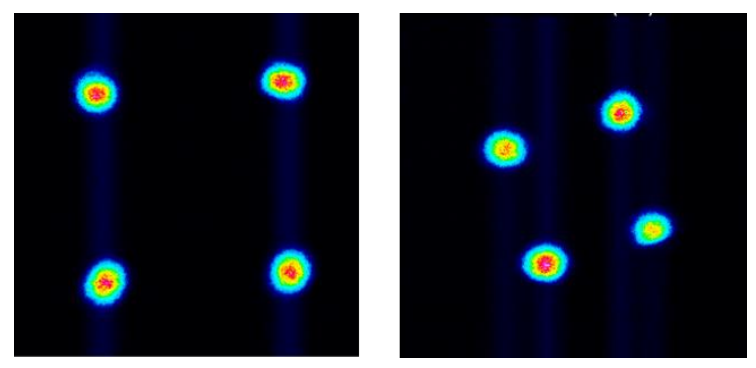

(a)

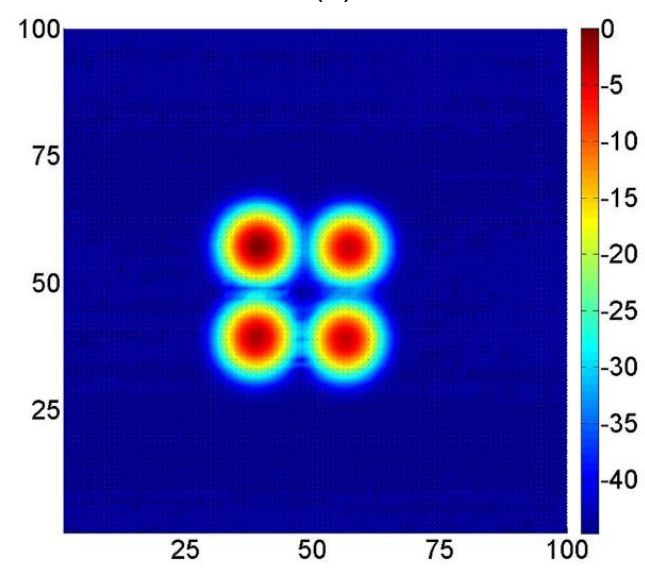

(b)

Fig. 2: (a) Beam profile before and after the FIFO device measured with a CCD and (b) output intensity distribution determined by a 2D power sampling method.

\section{Characterization of 4c-MCF FIFO}

To characterize the fabricated FIFO, 4 SMFs were evenly illuminated by a $1 \times 4$ PLC splitter from a single $1550 \mathrm{~nm}$ laser diode and the spatial intensity distribution before and after the FIFO was investigated with a CCD for initial 
comparison (Fig. 2(a)) and a power sampling method (Fig. 2(b)) was then used for quantitative investigation. As shown in Fig. 2(a), the input and output beam profiles stay almost the same and 4 clean spatial beams emerging from the $4 \mathrm{c}-\mathrm{MCF}$ were clearly observed. The average insertion loss was $\sim 2.8 \mathrm{~dB}$ and the core-to-core variation was less than $0.5 \mathrm{~dB}$. The insertion loss is mainly due to the MFD mismatch after demagnification and could be improved in the future by optimizing the demagnification ratio and/or enlarging the MFD of the input fibres. Next, the crosstalk (XT) of the fabricated FIFO was examined by launching the light into one of the input SMFs. As shown by the contour map in Fig. 3, the inter-core XT between the illuminated core and the neighbouring cores was less than $-42 \mathrm{~dB}(-42.6$, -44.5 and $-43.4 \mathrm{~dB}$, respectively).

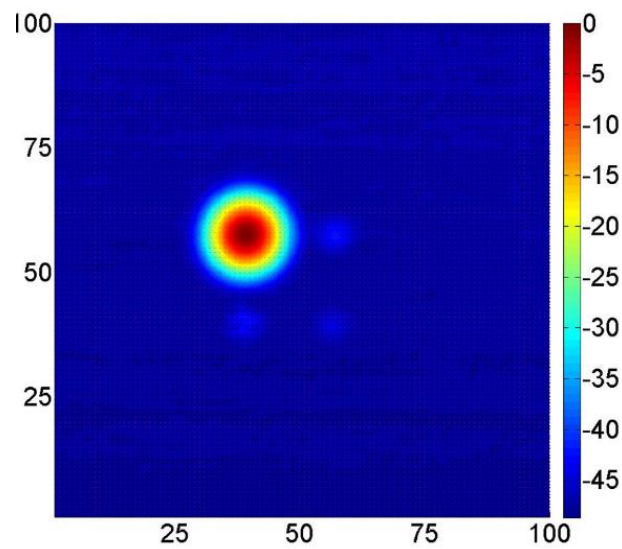

Fig. 3: Measured inter-core crosstalk contour map from the fabricated FIFO.

The insertion loss and XT of each core of the fabricated 4c-MCF FIFO are listed in Table 1. The insertion loss ranges from $2.6 \mathrm{~dB}$ to $3.1 \mathrm{~dB}$ and the worst core XT was less than $-40.2 \mathrm{~dB}$ at 1550 $\mathrm{nm}$. For comparison, we also have fabricated $3 \mathrm{D}$ waveguide based FIFO device and tested the optical performance. The average insertion loss was $\sim 1.5 \mathrm{~dB}$ with a core-to-core variation of 0.5 $\mathrm{dB}$ but the worst core XT was measured to be $33 \mathrm{~dB}$. This results indicate that the proposed fibre optic collimator based FIFO device can provide lower XT for high density MCF application. As a next step, we will optimize the insertion loss of the proposed device by using thin cladding fibres (to reduce the input array pitch) and will explore the scalability of the number of cores. Also, angled cleaves or antireflection coatings can be applied to the endfacet of the optical fibres to suppress the unwanted Fresnel back-reflections. There is enough free space region ( $20 \mathrm{~mm}$ ) between two collimators to allow the incorporation of many functional optical elements (e.g. isolators, filters etc.) which can enable compact and simple platform for the development of integrated multiport devices.

Tab. 1: Insertion loss and worst XT summary of the fabricated FIFO

\begin{tabular}{|c|c|c|}
\hline Ports & IL [dB] & Worst XT [dB] \\
\hline$\# 1$ & 3.1 & -42.5 \\
\hline$\# 2$ & 2.6 & -42.6 \\
\hline$\# 3$ & 3 & -40.2 \\
\hline$\# 4$ & 2.6 & -40.3 \\
\hline
\end{tabular}

\section{Conclusions}

A new MCF fan-in/fan-out device concept has been demonstrated using well-established fibre optic collimator assemblies. In a 4f-lens (two lens imaging) system, the core pitch distance of an input fibre array can be efficiently converted to match the core pitch of the output MCF and a 4core MCF FIFO has been successfully fabricated to demonstrate the proof-of-concept. The proposed technique can be used for most MCFs having either square or hexagonal core arrangements and has the potential to lead to high spatial density MCF FIFO devices with low crosstalk for future SDM transmission.

\section{Acknowledgements}

This project was supported by the EU-Japan coordinated R\&D project on "Scalable And Flexible optical Architecture for Reconfigurable Infrastructure (SAFARI)" commissioned by the Ministry of Internal Affairs and Communications (MIC) of Japan and EC Horizon 2020. This work is funded in part by the EU FP7 project COSIGN (FP7-ICT-2013.1.1, grant agreement number 619572).

\section{References}

[1] D. J. Richardson et al., "Space division multiplexing in optical fibres", Nat. Photon. 7, 354 (2013).

[2] V. Sleiffer et al., "73.7 Tb's $(06 \times 3 \times 256 \mathrm{~Gb} / \mathrm{s})$ mode division multiplexed DP-16QAM transmission with inline MM-EDFA," Opt. Exp. 20, B428 (2012).

[3] Y. Jung et al., "Fist demonstration and detailed characterization of a multimode amplifier for space division multiplexed transmission system", Opt. Exp. 19, B952 (2011).

[4] B. Zhu et al., "Seven-core multicore fiber transmissions for passive optical network" Opt. Exp. 18, 11117 (2010).

[5] R. R. Thomson et al., "Ultrafast laser inscription of a three dimentional fan-out device for multicore fiber coupling applications" Opt. Exp. 15, 11691 (2007).

[6] Y. Tottori et al., "Low loss optical connection module for seven-core multicore fiber and seven single mode fibers" IEEE Photon.Technol. Lett. 24, 1926 (2012).

[7] Y. Jung et al., "Compact few-mode fiber collimator and associated optical components for mode division multiplexed transmission" Proc. OFC, W2A.40 (2016).

[8] Y. Jung et al., "Compact 32-core multicore fibre isolator for high density spatial division multiplexed transmission" Proc. ECOC, W2.B4 (2016).

[9] Y. Jung et al., "All-fiber optical interconnection for dissimilar multicore fibers with low insertion loss", Proc. OFC, W3H.2 (2017) 